\title{
Q-factor dependent Kerr switching of semiconductor microcavities
}

\author{
Emre Yüce $^{1,2}$, Georgios Ctistis ${ }^{1,2}$, Julien Claudon ${ }^{3}$, Maela Bazin $^{3}$, Jean-Michel Gérard ${ }^{3}$ and Willem L. Vos ${ }^{1,2}$ \\ 1. Complex Photonic Systems (COPS), MESA+ Institute for Nanotechnology, \\ University of Twente, 7500 AE Enschede, The Netherlands \\ 2. Center for Nanophotonics FOM-Institute for Atomic and Molecular Physics (AMOLF) \\ Science park 113, 1098 XG Amsterdam. The Netherlands \\ 3. CEA/INAC/SP2M, Nanophysics and Semiconductor Laboratory, 38054 Grenoble, France
}

The need for low-cost, high-speed, and low power consumption photonic devices for communication applications has stimulated a significant amount of research in integrated photonics [1]. The light, which is confined by recirculation in a very small volume, can be manipulated in order to achieve high-speed data communication [2].

We present our results on GaAs-AlAs planar cavities with a cavity resonance in the telecom range and with different quality factors. Here, we show that a GaAs-AlAs microcavity with a quality factor $\mathrm{Q}=320$ can be switched within $1 \mathrm{ps}$ via the electronic Kerr effect. We observe that the speed of the switching time is only limited by the dynamics of the light in our cavity but not by the constituent materials. Figure 1(a) shows the ultimate fast change of the cavity resonance frequency versus the time delay between pump and probe beams. The largest shift in the resonance frequency is observed at zero delay, where pump and probe beam coincide in time.
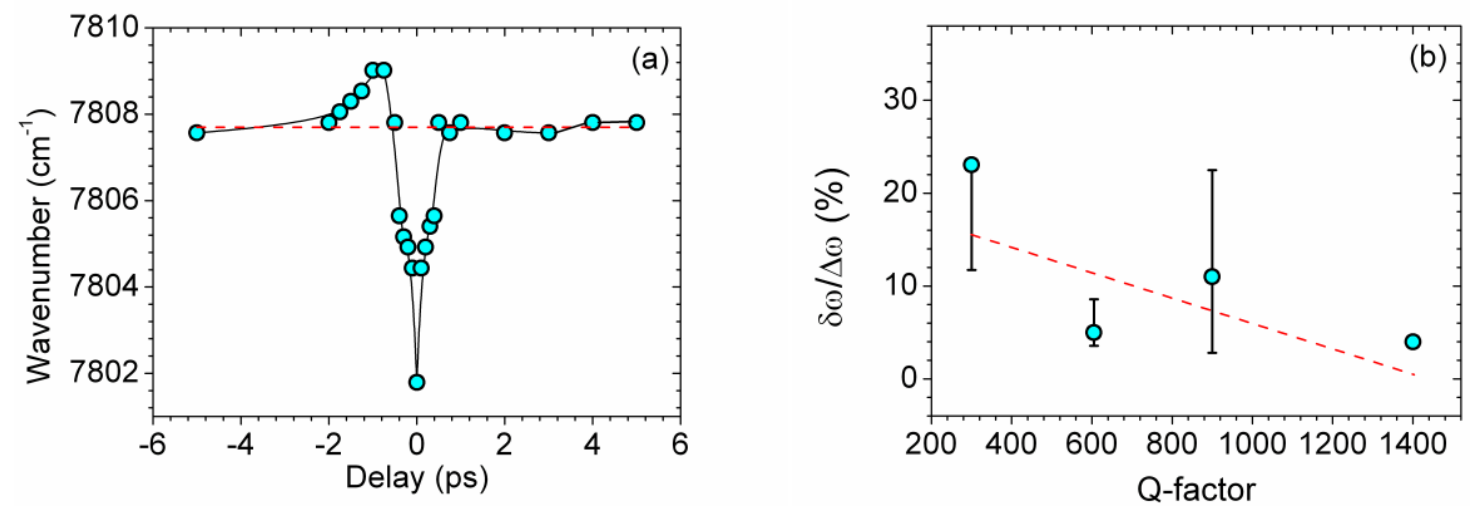

Fig. 1 (a) Frequency vs time delay between pump and probe for a cavity with $Q=320$. The red dashed line shows the cold cavity resonance frequency. (b) Relative frequency shift of the cavity resonance vs the quality factor. All measurements are carried out at the same pump intensity $\left(72.8 \mathrm{GW} / \mathrm{cm}^{2}\right)$.

We observe that the relative shift of the cavity resonance frequency $(\delta \omega)$ with respect to cavity line-width $(\Delta \omega)$ decreases with increasing quality factor. Figure 1(b) shows the relative resonance frequency shift versus the quality factor. All measurements are carried out at a constant pump intensity of $72.8 \mathrm{GW} / \mathrm{cm}^{2}$. Increasing the storage time of the cavity not only decreases the switching speed but also decreases the induced refractive index change. The electronic Kerr effect is maximized when the temporal and spatial overlap of pump and probe light is achieved. Increasing quality factor of the cavity results in a longer storage time for the probe light in the cavity and therefore a decrease in the temporal overlap of the pump and probe. As a result the shift of the resonance frequency decreases with increasing quality factor.

\section{References}

[1] E. Yüce, O. Gürlü, and A. Serpengüzel, IEEE Photon. Technol. Lett., 21, 1481 (2009).

[2] T. Tanabe, M. Notomi, H. Taniyama, and E. Kuramochi, Phys. Rev. Lett. 102, 043907 (2009). 